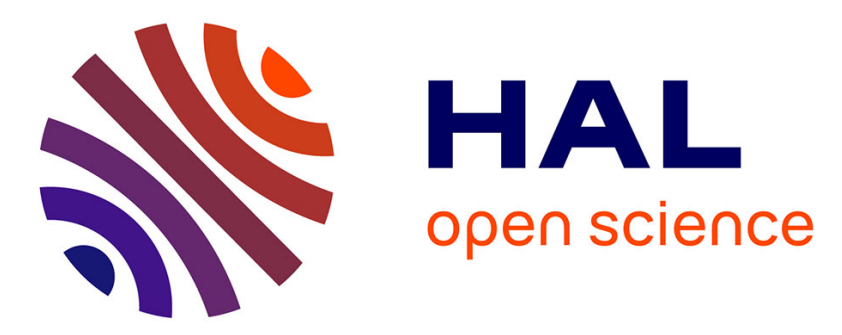

\title{
High-gain observers with limited gain power for systems with observability canonical form
}

Lei Wang, Daniele Astolfi, Lorenzo Marconi, Hongye Su

\section{To cite this version:}

Lei Wang, Daniele Astolfi, Lorenzo Marconi, Hongye Su. High-gain observers with limited gain power for systems with observability canonical form. Automatica, 2017, 75, pp.16-23. 10.1016/j.automatica.2016.09.006 . hal-02096457

\section{HAL Id: hal-02096457 \\ https://hal.science/hal-02096457}

Submitted on 16 May 2019

HAL is a multi-disciplinary open access archive for the deposit and dissemination of scientific research documents, whether they are published or not. The documents may come from teaching and research institutions in France or abroad, or from public or private research centers.
L'archive ouverte pluridisciplinaire HAL, est destinée au dépôt et à la diffusion de documents scientifiques de niveau recherche, publiés ou non, émanant des établissements d'enseignement et de recherche français ou étrangers, des laboratoires publics ou privés. 


\title{
High-gain observers with limited gain power for systems with observability canonical form
}

\author{
Lei Wang ${ }^{\mathrm{a}, \mathrm{b}}$, Daniele Astolfi ${ }^{\mathrm{b}, \mathrm{c}}$, Hongye $\mathrm{Su}^{\mathrm{a}}{ }^{\text {, Lorenzo Marconi }}{ }^{\mathrm{b}}$

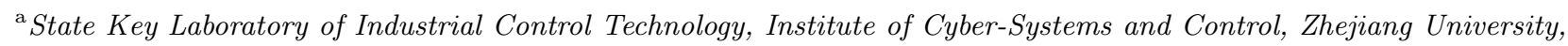 \\ Hangzhou, China. \\ b $C A S Y-D E I$, University of Bologna, Italy \\ c MINES ParisTech, PSL Research University, CAS - Centre automatique et systèmes, Paris, France
}

\begin{abstract}
We consider the problem of state observation for systems having a well-defined observability canonical form ([9]) by means of high-gain observers. The main goal is to show that, for this class of systems, observers can be designed with the highgain parameter powered just up to the order 2 regardless the dimension of the state system. In this way we substantially overtake the main limitations of standard design procedures in which the high-gain parameter is powered up to the order of the system. The observer structure, which generalises the ideas presented in [2], can be used in all those contexts where fast state observation is required, such as in the design of output feedback stabilisers by means of the nonlinear separation principle that is also specifically addressed in the paper.
\end{abstract}

Key words: Observability canonical form, high-gain observers, nonlinear separation principle.

\section{Introduction}

The problem of designing asymptotic state observers for nonlinear systems is a central topic in the control literature (see [6] and [9] for general surveys on the topic). A special role in literature is played by the so-called high-gain observer in which the error trajectory has an exponential decay rate that can be imposed arbitrarily fast by acting on a design parameter, appearing in the observer structure, typically known as "high-gain" parameter (see for instance the surveys $[15,16]$ and references therein). Such observers are routinely used in all control contexts where fast observation is mandatory, such as contexts of nonlinear output feedback stabilisation by means of the nonlinear separation principle in which fast observation is required in order to prevent finite escape times of the closed-loop system (see [22]). A very general and elegant framework where high-gain observers have been developed is the one presented in [9] where Luenberger style observers are designed for the class of nonlinear systems

Email addresses: 1wang@iipc.zju.edu.cn (Lei Wang), astolfi.daniele@gmail.com (Daniele Astolfi), hysu@iipc.zju.edu.cn (Hongye Su),

lorenzo.marconi@unibo.it (Lorenzo Marconi). that can be transformed, by change of variables, in the so-called observability canonical form. The latter is a special triangular form in which the partial derivatives of the functions describing the dynamics of the single state components with respect to the "first variable" never vanish. In the same book the existence of such normal forms is linked to a notion of observability for nonlinear systems (see also [10]). It is also shown how the proposed Luenberger style high-gain observer can be used, in a nonlinear separation principle context, to systematically design output feedback stabilisers. The application of high-gain observers in the fields of output regulation can be found in [18].

"Dirty derivatives observers" are further fundamental examples of high-gain structures used in several control contexts (see for instance [23]). In this case the goal is to obtain an arbitrarily accurate (i.e. practical) and arbitrarily fast estimate of the output of a nonlinear system and its time derivatives. Such "rough" observers have been shown to play a role in the context of output feedback stabilisation. In this respect it is worth quoting the milestone contribution in [7], which opened the door to a number of works on the subject (among which it is worth recalling $[24,14,19,11])$ culminated in the fundamental paper [23]. In the latter the use of dirty derivatives observers have been proved to be effective 
to systematically stabilize, by output feedback, a broad class of nonlinear systems stabilizable by uniformly completely observable (UCO) state feedback control laws. The main drawbacks of observer design techniques that rely on high-gain properties are typically two-fold. The first is known as "peaking phenomenon", whose effect in many control contexts is overtaken by using saturation functions $[14,22]$. The second is related to the fact that the high-gain parameter is typically powered to the order given by the dimension of the observed system. This fact, in turn, makes the practical (i.e. numerical) implementation of such observers very hard whenever the order of the observed system is high. A further consequence of this, is that the sensitivity to high-frequency measurement noise of these observers is typically unacceptable, as already studied in $[1,5,25]$.

In the recent contribution [2], a new high-gain observer structure has been proposed for a class of uniformly observable nonlinear systems which are diffeomorphic to the canonical observability form [10]. The remarkable feature of the new observers is that the high-gain parameter is powered up to the order 2, regardless the dimension of the observed system, at the price of having the observer state dimension $2 n-2$ with $n$ the dimension of the observed system. The new observer has been shown to substantially overtake the problems related to numerical computation. Moreover it has been shown that, in the linear case, this observer has better asymptotic properties with respect to high-frequency noise. In this paper, we extend the ideas and arguments of [2] to a wider class of observable nonlinear systems. In particular we consider nonlinear systems, possibly not affine in the input, which are Uniformly Observable (according to the definition given in ([9, Section 2, Definition 2.1]) and therefore diffeomorphic to a observability canonical form, [9, Section 3, Theorem 2.1], which is more general than the one considered in [2]. As in [2], the new observer structure overtakes the problem of numerical implementation of the classical observer for high order systems and substantially improves the observation performances in terms of sensitivity to high-frequency measurement noise.

The paper is organized as follows. In Section 2 the framework is introduced by presenting existing results on Luenberger style observers for observability canonical form. The main result is given in Section 3, where the new high-gain observer is proposed and its convergence analysis is derived. Then its application to a nonlinear separation principle is discussed in Section 4. In Section 5 the performances of the new observer are shown in a numerical example. Finally, Section 6 present final remarks.

\section{The Framework}

In this paper we deal with single-input single-output nonlinear systems of the form

$$
\dot{x}=\psi(x, u), \quad y=\rho(x, u)
$$

where $x \in \mathbb{R}^{n}$ is the state, $u \in U \subset \mathbb{R}$ is the control input, $y \in \mathbb{R}$ is the measured output, $\psi(x, u)$ are smooth vector fields, and $\rho(x, u)$ is a smooth function. The main goal of the paper is to develop a new observer for the previous class of systems to be used in a context of nonlinear separation principle. The reference framework that is used to present the result is the one of [9] in which Luenberger style high-gain observers are proposed. In that framework, in particular, the interest is on systems that are Uniformly Observable, meaning that there exists a global change of variables $z=\Phi(x)$ that transforms system (1) into the so-called observability canonical form

$$
\begin{aligned}
\dot{z}_{i} & =f_{i}\left(\mathbf{z}_{i}, z_{i+1}, u\right), \quad 1 \leq i \leq n-1 \\
\dot{z}_{n} & =f_{n}\left(\mathbf{z}_{n}, u\right) \\
y & =h\left(z_{1}, u\right)
\end{aligned}
$$

with $z=\left(z_{1}, \ldots, z_{n}\right)^{T}, \mathbf{z}_{i}=\left(z_{1}, \ldots, z_{i}\right)^{T}$ and with the functions $f_{i}(\cdot), i=1, \ldots, n-1$ and $h(\cdot)$ that, for any $(z, u) \in \mathbb{R}^{n} \times U$, fulfil

$\frac{\partial h}{\partial z_{1}}\left(z_{1}, u\right) \neq 0, \frac{\partial f_{i}}{\partial z_{i+1}}\left(\mathbf{z}_{i}, z_{i+1}, u\right) \neq 0, i=1, \ldots, n-1$.

Furthermore, we assume that system (2) satisfies the following two assumptions.

Assumption 1 The maps $f_{i}(\cdot), i=1, \ldots, n$, are globally Lipschitz with respect to $\mathbf{z}_{i}$, uniformly with respect to $u$ and $z_{i+1}$, namely there exists a $\ell>0$ such that for all $\mathbf{z}_{i} \in \mathbb{R}^{i}, \mathbf{z}_{i}^{\prime} \in \mathbb{R}^{i}, z_{i+1} \in \mathbb{R}$, and $u \in U$ the following holds

$$
\begin{aligned}
&\left|f_{i}\left(\mathbf{z}_{i}, z_{i+1}, u\right)-f_{i}\left(\mathbf{z}_{i}^{\prime}, z_{i+1}, u\right)\right| \leq \ell\left|\mathbf{z}_{i}-\mathbf{z}_{i}^{\prime}\right| \\
& 1 \leq i \leq n-1, \\
&\left|f_{n}\left(\mathbf{z}_{n}, u\right)-f_{n}\left(\mathbf{z}_{n}^{\prime}, u\right)\right| \leq \ell\left|\mathbf{z}_{n}-\mathbf{z}_{n}^{\prime}\right|
\end{aligned}
$$

Assumption 2 There exist two positive real constants $\alpha<\beta$, such that for all $(z, u) \in \mathbb{R}^{n} \times U$ the following holds

$$
\begin{gathered}
\alpha \leq\left|\frac{\partial h\left(z_{1}, u\right)}{\partial z_{1}}\right| \leq \beta \\
\alpha \leq\left|\frac{\partial f_{i}\left(\mathbf{z}_{i}, z_{i+1}, u\right)}{\partial z_{i+1}}\right| \leq \beta, \quad 1 \leq i \leq n-1 .
\end{gathered}
$$

The globally Lipschitz condition in Assumption 1 is motivated by the fact that, in the following, we look for a global observer. In case just semiglobal observation is looked for, namely if the initial conditions of the observer and of the system range in a fixed known compact set, the previous condition can be weakened by asking that 
the functions $f_{i}(\cdot)$ are only locally Lipschitz with respect to $\mathbf{z}_{\mathbf{i}}($ see $[10])$.

Within this framework the main result proposed in $[9$, Theorem 2.2 , Chapter 6$]$ is a systematic design of a high-gain Luenberger style observer that takes the form

$$
\begin{aligned}
& \dot{\hat{z}}_{i}=f_{i}\left(\hat{\mathbf{z}}_{i}, \hat{z}_{i+1}, u\right)+\kappa^{i} k_{i}\left(y-h\left(\hat{z}_{1}, u\right)\right) \\
& \quad 1 \leq i \leq n-1, \\
& \dot{\hat{z}}_{n}=f_{n}\left(\hat{\mathbf{z}}_{n}, u\right)+\kappa^{n} k_{n}\left(y-h\left(\hat{z}_{1}, u\right)\right)
\end{aligned}
$$

where $\hat{z}=\left(\hat{z}_{1}, \ldots, \hat{z}_{n}\right)^{T}, \hat{\mathbf{z}}_{i}=\left(\hat{z}_{1}, \ldots, \hat{z}_{i}\right)^{T}, k_{i}$ for $i=$ $1, \ldots, n$ are appropriate coefficients and $\kappa$ is a positive high-gain parameter. As a matter result the following result holds (see [9]).

Theorem 1 Consider the observed system (2) and the observer (4) under Assumptions 1 and 2. There exist a choice of $k_{1}, \ldots, k_{n}$ and $\kappa^{\star} \geq 1$ such that for all $\kappa>\kappa^{\star}$ the following bound holds

$$
|z(t)-\hat{z}(t)| \leq \mu_{1} \kappa^{n-1} \exp \left(-\mu_{2} \kappa t\right)|z(0)-\hat{z}(0)|
$$

for all $(z(0), \hat{z}(0)) \in \mathbb{R}^{n} \times \mathbb{R}^{n}$, for all $t \geq 0$, for some positive $\mu_{1}$ and $\mu_{2}$ independent of $\kappa$.

One of the drawbacks of observers of the form (4) is clearly related to the increasing power (up to the order $n$ ) of the high-gain parameter $\kappa$, which makes the practical numerical implementation an hard task when $n$ is very large. Motivated by these considerations, we propose in the next section a new observer for the class of uniformly observable systems that preserves the same high-gain features of the "classical" observer by substantially overtaking the implementation problems mentioned before. Specifically, we present a high-gain observer structure with a gain which grows only up to power 2 (regardless the dimension $n$ of the system), at the price of having the observer state dimension $2 n-2$. This is done by extending the ideas and the arguments proposed in [2] in which the problem of state observation of systems in the canonical observability form

$$
\dot{z}_{1}=z_{2}, \quad \cdots \quad \dot{z}_{n-1}=z_{n}, \quad \dot{z}_{n}=\varphi(z)
$$

with $\varphi(\cdot)$ a locally Lipschitz function, is presented. That paper, in fact, proposed a $(2 n-2)$-dimensional observer of the form

$$
\begin{aligned}
\dot{\zeta}_{i} & =\left(\begin{array}{c}
B^{T} \zeta_{i}-\kappa k_{i 1} e_{i} \\
B^{T} \zeta_{i+1}-\kappa^{2} k_{i 2} e_{i}
\end{array}\right), 1 \leq i \leq n-2, \\
\dot{\zeta}_{n-1} & =\left(\begin{array}{c}
B^{T} \zeta_{n-1}-\kappa k_{n-1,1} e_{n-1} \\
\varphi_{s}\left(\hat{z}^{\prime}\right)-\kappa^{2} k_{n-1,2} e_{n-1}
\end{array}\right),
\end{aligned}
$$

in which $\zeta_{i} \in \mathbb{R}^{2}, i=1, \ldots, n-1, e_{1}:=C \zeta_{i}-y$,

$$
e_{i}:=C \zeta_{i}-B^{T} \zeta_{i-1}, \quad i=2, \ldots, n-1,
$$

with $C:=\left(\begin{array}{ll}1 & 0\end{array}\right)$ and $B^{T}:=\left(\begin{array}{ll}0 & 1\end{array}\right), K_{i}:=\left(k_{i 1}, k_{i 2}\right)^{\top}$ are design parameters,

$$
\begin{gathered}
\hat{z}^{\prime}:=L_{1} \xi, \quad L_{1}:=\operatorname{blkdiag}(\underbrace{C, \ldots, C}_{(n-2) \text { times }}, I_{2}), \\
\zeta:=\operatorname{col}\left(\zeta_{1}, \ldots, \zeta_{n-1}\right) \in \mathbb{R}^{2 n-2},
\end{gathered}
$$

and $\varphi_{s}(\cdot)$ is an appropriate saturated version of $\varphi(\cdot)$. In turn, if the design parameters $K_{i}, i=1, \ldots, n-1$, are designed as shown in Lemma 1 of [2] and $\kappa$ is taken sufficiently large according to the Lipschitz constant of $\varphi(\cdot)$, the variable $\hat{z}$ converges asymptotically to $z$ with a convergence rate that can be arbitrarily decreased by increasing $\kappa$ (see Proposition 1 in [2]).

\section{The new observer design}

Instrumental to the main result is the following lemma that refers to the block-tridiagonal matrix $M(t) \in \mathbb{R}^{(2 n-2) \times(2 n-2)}$ defined as

$M(t)=\left(\begin{array}{cccccc}E_{1}(t) & N_{2}(t) & 0 & \ldots & \ldots & 0 \\ Q_{2} & E_{2}(t) & N_{3}(t) & \ddots & & \vdots \\ 0 & \ddots & \ddots & \ddots & \ddots & \vdots \\ \vdots & \ddots & \ddots & \ddots & \ddots & 0 \\ \vdots & & \ddots & Q_{n-2} & E_{n-2}(t) & N_{n-1}(t) \\ 0 & \ldots & \ldots & 0 & Q_{n-1} & E_{n-1}(t)\end{array}\right)$

with $E_{i}(t) \in \mathbb{R}^{2 \times 2}, Q_{i} \in \mathbb{R}^{2 \times 2}$, and $N_{i}(t) \in \mathbb{R}^{2 \times 2}$ matrices defined as

$$
\begin{aligned}
& E_{i}(t)=\left(\begin{array}{cc}
-k_{i 1} b_{i}(t) & a_{i}(t) \\
-k_{i 2} b_{i}(t) & 0
\end{array}\right), \quad Q_{i}=\left(\begin{array}{ll}
0 & k_{i 1} \\
0 & k_{i 2}
\end{array}\right), \\
& N_{i}(t)=\left(\begin{array}{cc}
0 & 0 \\
0 & a_{i}(t)
\end{array}\right), \quad i=1, \ldots, n-1
\end{aligned}
$$

where $a_{i}(t)$ and $b_{i}(t)$ are positive ${ }^{1}$ for all $1 \leq i \leq n-1$ and $t \geq 0$, and $\left(k_{i 1}, k_{i 2}\right)$ are positive coefficients. The proof of the forthcoming lemma is given in Appendix-A.

Lemma 1 Consider the matrix $M(t)$ in (9) with $a_{i}(t)$ and $b_{i}(t)$ fulfilling $\alpha \leq a_{i}(t) \leq \beta, \alpha \leq b_{i}(t) \leq \beta$ for

\footnotetext{
${ }^{1}$ All the forthcoming analysis can be easily adapted to deal with the case in which (some of) the $a_{i}$ s and $b_{i}$ s are negative.
} 
some positive $\alpha$ and $\beta$ for all $1 \leq i \leq n-1$ and $t \geq 0$. Then there exist coefficients $\left(k_{i 1}, k_{i 2}\right), i=1, \ldots, n-1$, a symmetric positive definite matrix $P$ and a positive constant $\lambda$, such that

$$
P M(t)+M(t)^{\top} P \leq-\lambda I
$$

for all $t \geq 0$.

It is worth noting that Lemma 1 extends the result in Lemma 2.1 in $[9$, Chapter 6$]$ in a non-trivial way due to the specific tri-block diagonal structure of $M(t)$ in (9). Furthermore, this result can be seen also as an extension to the time-varying case of the result in [2, Lemma 1], in which $a_{i}(t)=b_{i}(t)=1$ for all $i=1, \ldots, n-1$ and for all $t \geq 0$.

The new observer has state $\zeta \in \mathbb{R}^{2 n-1}$ defined in (8) with the dynamics of $\zeta_{i} \in \mathbb{R}^{2}, i=1, \ldots, n-1$, given by (compare with (5))

$$
\begin{aligned}
& \dot{\zeta}_{i}=\left(\begin{array}{c}
f_{i}\left(\hat{\mathbf{z}}_{i}, B^{T} \zeta_{i}, u\right)-\kappa k_{i 1} e_{i} \\
f_{i+1}\left(\hat{\mathbf{z}}_{i+1}, B^{T} \zeta_{i+1}, u\right)-\kappa^{2} k_{i 2} e_{i}
\end{array}\right), \\
& 1 \leq i \leq n-2, \\
& \dot{\zeta}_{n-1}=\left(\begin{array}{c}
f_{n-1}\left(\hat{\mathbf{z}}_{n-1}, B^{T} \zeta_{n-1}, u\right)-\kappa k_{n-1,1} e_{n-1} \\
f_{n}\left(\hat{\mathbf{z}}_{n}, u\right)-\kappa^{2} k_{n-1,2} e_{n-1}
\end{array}\right),
\end{aligned}
$$

in which $B$ and $C$ are defined as in the previous section, $e_{1}:=h\left(C \zeta_{1}, u\right)-y, e_{i}, i=2, \ldots, n-1$, are defined as in $(6), K_{i}=\left(k_{i 1}, k_{i 2}\right)^{\top}$ are coefficients to be chosen according to the previous Lemma 1 , and $\kappa$ is the highgain parameter. In (11), the vectors $\hat{\mathbf{z}}_{i}:=\left(\hat{z}_{1}, \ldots, \hat{z}_{i}\right)^{T}$, $i=1, \ldots, n$, contain estimates of the first $i$ components of the state $z$ that can be extracted by the observer state $\zeta$. In particular, as in [2], the redundancy of the observer can be employed to obtain two different estimates $\hat{z}=$ $\left(\hat{z}_{1}, \ldots, \hat{z}_{n}\right)^{T}$ of the state variable $z$ that can be equally used to define $\hat{\mathbf{z}}_{i}$. The first, denoted by $\hat{z}^{\prime}$, is defined as in (7). The second, denoted by $\hat{z}^{\prime \prime}$, is described by

$$
\hat{z}^{\prime \prime}:=L_{2} \zeta, \quad L_{2}:=\operatorname{blkdiag}(I_{2}, \underbrace{B^{\top}, \ldots, B^{\top}}_{(n-2) \text { times }}) .
$$

Similarly to the result in [2], in the forthcoming theorem we show that the variables $\hat{z}^{\prime}$ and $\hat{z}^{\prime \prime}$ asymptotically recover the value of the state of $(2)$ if the coefficients $\left(k_{i 1}, k_{i 2}\right), i=1, \ldots, n-1$ are properly chosen and $\kappa$ is large enough. In the statement of the proposition we denote

$$
\hat{Z}=\operatorname{col}\left(\hat{z}^{\prime}, \hat{z}^{\prime \prime}\right), \quad Z=\operatorname{col}(z, z) .
$$

Theorem 2 Consider the system (2) satisfying Assumptions 1 and 2, and the observer (11) with the coefficients $\left(k_{i 1}, k_{i 2}\right)$ chosen according to Lemma 1 with $\alpha$ and $\beta$ given by Assumption 2 for some $P=P^{\top}>0$ and $\lambda>0$. Then, there exist $a \kappa^{\star} \geq 1$ and positive constants $c_{1}, c_{2}$, such that, for all $\kappa>\kappa^{\star}$ the following bound holds

$$
|\hat{Z}(t)-Z(t)| \leq c_{1} \kappa^{n-1} \exp \left(-c_{2} \kappa t\right)|\hat{Z}(0)-Z(0)|
$$

for all $(z(0), \zeta(0)) \in \mathbb{R}^{n} \times \mathbb{R}^{2 n-2}$ and for all $t \geq 0$.

Proof. Consider the change of variable

$\zeta_{i} \mapsto \tilde{\zeta}_{i}:=\zeta_{i}-\operatorname{col}\left(z_{i}, z_{i+1}\right)=\operatorname{col}\left(\zeta_{i 1}-z_{i}, \zeta_{i 2}-z_{i+1}\right)$,

where the $\zeta_{i}=\operatorname{col}\left(\zeta_{i 1}, \zeta_{i 2}\right)$. As far as the $\tilde{\zeta}_{1}$ dynamics are concerned, by using the mean value theorem, it turns out that

$$
\begin{aligned}
& \dot{\tilde{\zeta}}_{11}=f_{1}\left(\hat{\mathbf{z}}_{1}, B^{T} \zeta_{1}, u\right)-f_{1}\left(\mathbf{z}_{1}, z_{2}, u\right)-\kappa k_{11} e_{1} \\
& =f_{1}\left(\hat{\mathbf{z}}_{1}, B^{T} \zeta_{1}, u\right)-f_{1}\left(\mathbf{z}_{1}, B^{T} \zeta_{1}, u\right)+f_{1}\left(\mathbf{z}_{1}, B^{T} \zeta_{1}, u\right) \\
& \quad-f_{1}\left(\mathbf{z}_{1}, z_{2}, u\right)-\kappa k_{11}\left(h\left(C \zeta_{1}, u\right)-h\left(z_{1}, u\right)\right) \\
& =\frac{\partial f_{1}}{\partial z_{2}}\left(\mathbf{z}_{1}(t), \delta_{1}(t), u(t)\right) \tilde{\zeta}_{12}-\kappa k_{11} \frac{\partial h}{\partial z_{1}}\left(\delta_{0}(t), u(t)\right) \tilde{\zeta}_{11} \\
& \quad+f_{1}\left(\hat{\mathbf{z}}_{1}, B^{\top} \zeta_{1}, u\right)-f_{1}\left(\mathbf{z}_{1}, B^{\top} \zeta_{1}, u\right) \\
& \dot{\tilde{\zeta}}_{12}=f_{2}\left(\hat{\mathbf{z}}_{2}, B^{\top} \zeta_{2}, u\right)-f_{2}\left(\mathbf{z}_{2}, z_{3}, u\right)-\kappa^{2} k_{12} e_{1} \\
& =f_{2}\left(\hat{\mathbf{z}}_{2}, B^{\top} \zeta_{2}, u\right)-f_{2}\left(\mathbf{z}_{2}, B^{\top} \zeta_{2}, u\right)+f_{2}\left(\mathbf{z}_{2}, B^{\top} \zeta_{2}, u\right) \\
& \quad-f_{2}\left(\mathbf{z}_{2}, z_{3}, u\right)-\kappa^{2} k_{12}\left(h\left(C \zeta_{1}, u\right)-h\left(z_{1}, u\right)\right) \\
& =\frac{\partial f_{2}}{\partial z_{3}}\left(\mathbf{z}_{2}(t), \delta_{2}(t), u(t)\right) \tilde{\zeta}_{22}-\kappa^{2} k_{12} \frac{\partial h}{\partial z_{1}}\left(\delta_{0}(t), u(t)\right) \tilde{\zeta}_{11} \\
& \quad+f_{2}\left(\hat{\mathbf{z}}_{2}, B^{\top} \zeta_{2}, u\right)-f_{2}\left(\mathbf{z}_{2}, B^{\top} \zeta_{2}, u\right)
\end{aligned}
$$

for some $\delta_{0}(t)$ and $\delta_{1}(t)$, namely, by setting

$$
\begin{aligned}
b_{1}(t) & :=\frac{\partial h}{\partial z_{1}}\left(\delta_{0}(t), u(t)\right) \\
a_{1}(t) & :=\frac{\partial f_{1}}{\partial z_{2}}\left(\mathbf{z}_{1}(t), \delta_{1}(t), u(t)\right) \\
a_{2}(t) & :=\frac{\partial f_{2}}{\partial z_{3}}\left(\mathbf{z}_{2}(t), \delta_{2}(t), u(t)\right)
\end{aligned}
$$

we obtain

$$
\begin{aligned}
& \dot{\tilde{\zeta}}_{11}=a_{1}(t) \tilde{\zeta}_{12}-\kappa k_{11} b_{1}(t) \tilde{\zeta}_{11}+\bar{f}_{1}(t) \\
& \dot{\tilde{\zeta}}_{12}=a_{2}(t) \tilde{\zeta}_{22}-\kappa^{2} k_{12} b_{1}(t) \tilde{\zeta}_{11}+\bar{f}_{2}(t)
\end{aligned}
$$

where

$$
\begin{aligned}
& \bar{f}_{1}(t):=f_{1}\left(\hat{\mathbf{z}}_{1}, B^{\top} \zeta_{1}, u\right)-f_{1}\left(\mathbf{z}_{1}, B^{\top} \zeta_{1}, u\right) \\
& \bar{f}_{2}(t):=f_{2}\left(\hat{\mathbf{z}}_{2}, B^{\top} \zeta_{2}, u\right)-f_{2}\left(\mathbf{z}_{2}, B^{\top} \zeta_{2}, u\right) .
\end{aligned}
$$

The $\tilde{\zeta}_{1}$ dynamics is thus described by

$$
\dot{\tilde{\zeta}}_{1}=H_{1}(t) \tilde{\zeta}_{1}+N_{2}(t) \tilde{\zeta}_{2}+\bar{F}_{1}(t)
$$


with

$H_{1}(t):=\left(\begin{array}{cc}-\kappa k_{11} b_{1}(t) & a_{1}(t) \\ -\kappa^{2} k_{12} b_{1}(t) & 0\end{array}\right), \quad \bar{F}_{1}(t):=\left(\begin{array}{c}\bar{f}_{1}(t) \\ \bar{f}_{2}(t)\end{array}\right)$,

and $N_{2}$ defined as in (9). Applying the same procedure to the $\tilde{\zeta}_{i}$ dynamics for $2 \leq i \leq n-2$, we obtain

$\dot{\tilde{\zeta}}_{i 1}=a_{i}(t) \tilde{\zeta}_{i 2}-\kappa k_{i 1} \tilde{\zeta}_{i 1}+\kappa k_{i 1} \tilde{\zeta}_{i-1,2}+\bar{f}_{i}(t)$,

$\dot{\tilde{\zeta}}_{i 2}=a_{i+1}(t) \tilde{\zeta}_{i+1,2}-\kappa^{2} k_{i 2} \tilde{\zeta}_{i 1}+\kappa^{2} k_{i 2} \tilde{\zeta}_{i-1,2}+\bar{f}_{i+1}(t)$,

where we have defined

$$
\begin{aligned}
& a_{i+1}(t):=\frac{\partial f_{i+1}}{\partial z_{i+2}}\left(\mathbf{z}_{i+1}(t), \delta_{i+1}(t), u(t)\right) \\
& \bar{f}_{i}(t):=f_{i}\left(\hat{\mathbf{z}}_{i}(t), B^{\top} \zeta_{i}(t), u(t)\right)-f_{i}\left(\mathbf{z}_{i}(t), B^{\top} \zeta_{i}(t), u(t)\right) \\
& \bar{f}_{i+1}(t):=f_{i+1}\left(\hat{\mathbf{z}}_{i+1}(t), B^{\top} \zeta_{i+1}(t), u(t)\right)- \\
& f_{i+1}\left(\mathbf{z}_{i+1}(t), B^{\top} \zeta_{i+1}(t), u(t)\right)
\end{aligned}
$$

for some $\delta_{i+1}(t)$. Thus, we get the $\tilde{\zeta}_{i}$ dynamics

$$
\dot{\tilde{\zeta}}_{i}=H_{i}(t) \tilde{\zeta}_{i}+N_{i+1}(t) \tilde{\zeta}_{i+1}+D_{2}(\kappa) Q_{i} \tilde{\zeta}_{i-1}+\bar{F}_{i}(t)
$$

with

$$
H_{i}(t):=\left(\begin{array}{cc}
-\kappa k_{i 1} & a_{i}(t) \\
-\kappa^{2} k_{i 2} & 0
\end{array}\right), \quad \bar{F}_{i}(t):=\left(\begin{array}{c}
\bar{f}_{i}(t) \\
\bar{f}_{i+1}(t)
\end{array}\right),
$$

$D_{2}(\kappa)=\operatorname{diag}\left(\kappa, \kappa^{2}\right)$ and $Q_{i}$ defined as in (9). Similarly the $\tilde{\zeta}_{n-1}$ is modelled by

$$
\begin{aligned}
& \dot{\tilde{\zeta}}_{n-1,1}=a_{n-1}(t) \tilde{\zeta}_{n-1,2}-\kappa k_{n-1,1} \tilde{\zeta}_{n-1,1} \\
& +\kappa k_{n-1,1} \tilde{\zeta}_{n-2,2}+\bar{f}_{n-1}(t), \\
& \dot{\tilde{\zeta}}_{n-1,2}=-\kappa^{2} k_{n-1,2} \tilde{\zeta}_{n-1,1}+\kappa^{2} k_{n-1,2} \tilde{\zeta}_{n-2,2}+\bar{f}_{n}(t),
\end{aligned}
$$

where we have defined

$$
\begin{aligned}
& \bar{f}_{n-1}(t):=f_{n-1}\left(\hat{\mathbf{z}}_{n-1}, B^{\top} \zeta_{n-1}, u\right) \\
&-f_{n-1}\left(\mathbf{z}_{n-1}, B^{\top} \zeta_{n-1}, u\right) \\
& \bar{f}_{n}(t):=f_{n}\left(\hat{\mathbf{z}}_{n}, u\right)-f_{n}\left(\mathbf{z}_{n}, u\right) .
\end{aligned}
$$

In more compact form the $\tilde{\zeta}_{n-1}$ dynamics can be rewritten as

$$
\dot{\tilde{\zeta}}_{n-1}=H_{n-1}(t) \tilde{\zeta}_{n-1}+D_{2}(\kappa) Q_{n-1} \tilde{\zeta}_{n-2}+\bar{F}_{n-1}(t),
$$

in which (by dropping the time-dependence for the sake of compactness)

$$
H_{n-1}:=\left(\begin{array}{cc}
-\kappa k_{n-1,1} & a_{n-1}(t) \\
-\kappa^{2} k_{n-1,2} & 0
\end{array}\right), \quad \bar{F}_{n-1}:=\left(\begin{array}{c}
\bar{f}_{n-1}(t) \\
\bar{f}_{n}(t)
\end{array}\right)
$$

Now note that, by Assumption 1,

$$
\left|\bar{f}_{i}(t)\right| \leq \ell\left|\tilde{\mathbf{z}}_{i}\right|, \quad 1 \leq i \leq n-1, \quad\left|\bar{f}_{n}(t)\right| \leq \ell|\tilde{\mathbf{z}}|,
$$

where, for convenience, we set $\tilde{\mathbf{z}}_{i}=\operatorname{col}\left(C \tilde{\zeta}_{1}, \ldots, C \tilde{\zeta}_{i}\right)$ for $1 \leq i \leq n-1$ and $\tilde{\mathbf{z}}=\operatorname{col}\left(\tilde{\mathbf{z}}_{n-1}, B^{\top} \tilde{\zeta}_{n-1}\right)$. Rescale now the variables $\tilde{\zeta}_{i}$ as follows

$$
\varepsilon_{i}:=\kappa^{2-i} D_{2}(\kappa)^{-1} \tilde{\zeta}_{i}, \quad i=1,2, \ldots, n-1
$$

By setting $\varepsilon=\operatorname{col}\left(\varepsilon_{1}, \ldots, \varepsilon_{n-1}\right)$, an easy calculation shows that

$$
\dot{\varepsilon}=\kappa M(t) \varepsilon+\bar{F}_{\kappa}(t)
$$

in which the matrix $M(t)$ is defined as (9), the terms $a_{i}(t)$, with $1 \leq i \leq n-1$, and $b_{1}(t)$ are bounded from below and from above for all $t \geq 0$ by Assumption 2, the term $b_{i}(t)=1$ for $2 \leq i \leq n-1$, and the vector $\bar{F}_{\kappa}(t)$ is defined by

$$
\bar{F}_{\kappa}(t):=\Delta_{\kappa} \bar{F}(t)
$$

with $\Delta_{\kappa}$ and $\bar{F}(t)$ defined by

$$
\begin{aligned}
\Delta_{\kappa} & :=\operatorname{diag}\left(\kappa, 1, \kappa^{-1}, \ldots, \kappa^{3-n}\right) \otimes D_{2}(\kappa)^{-1} \\
\bar{F}(t) & :=\operatorname{col}\left(\bar{F}_{1}, \bar{F}_{2}(t), \ldots, \bar{F}_{n-1}(t)\right) .
\end{aligned}
$$

Inspection on the each element of $\bar{F}_{\kappa}$ shows that, for $\kappa>1$,

$$
\left|\kappa^{1-i} \bar{f}_{i}(t)\right| \leq \ell|\varepsilon|, \quad i=1,2, \ldots, n,
$$

thus yielding that there exists a real number $\bar{\ell}>0$, independent of $\kappa$, such that $\left|\bar{F}_{\kappa}\right| \leq \bar{\ell}|\varepsilon|$. Now let the coefficients $\left(k_{i 1}, k_{i 2}\right)$ be chosen following Lemma 1 (see Appendix A) for a given symmetric and positive definite matrix $P$ and positive constant $\lambda$, and choose the Lyapunov candidate as $W(\varepsilon)=\varepsilon^{\top} P \varepsilon$. The time derivative of $W(\varepsilon)$ along the trajectories of system (15) is given by

$$
\begin{aligned}
\dot{W}(\varepsilon) & =\kappa \varepsilon^{\top}\left(P M+M^{\top} P\right) \varepsilon+2 \varepsilon^{\top} P \bar{F}_{\kappa} \\
& \leq-(\kappa \lambda-2 \bar{\ell}\|P\|)|\varepsilon|^{2} .
\end{aligned}
$$

Choosing $\kappa^{\star}=\frac{2 \bar{\ell}\|P\|}{\lambda}$, one can conclude that, for any $\kappa>\kappa^{\star}$, there exists a positive constant $\alpha_{1}$ such that $\dot{W} \leq-\alpha_{1} \kappa|\varepsilon|^{2}$. Recalling the fact that there exist positive constants $\bar{\sigma}$ and $\underline{\sigma}$ such that $\underline{\sigma}|\varepsilon|^{2} \leq W(\varepsilon) \leq \bar{\sigma}|\varepsilon|^{2}$, it can be further deduced that

$$
|\varepsilon(t)| \leq \bar{c}_{1} \exp \left(-\bar{c}_{2} \kappa t\right)|\varepsilon(0)|,
$$


for some proper positive constants $\bar{c}_{1}$ and $\bar{c}_{2}$, independent of $\kappa$. Now using the fact that, for all $\kappa>1$, $\kappa^{-(n-1)}|\tilde{\zeta}| \leq|\varepsilon| \leq|\tilde{\zeta}|$ and $|\tilde{\zeta}| \leq|\hat{Z}-Z| \leq 2|\tilde{\zeta}|$, the previous bound leads to the result.

It is worth noting that a key feature of the new observer is that the relative degree between the input $y$ (output of the observed system) and the error variables $\tilde{\zeta}_{i}$ defined in (12) is equal to one for $i=1$ and, in the worst case in which the functions $f_{i}(\cdot)$ depends on all the state variables in $\mathbf{z}_{\mathbf{i}}$, to two for all $i=2, \ldots, n$. This is due to the fact that the innovation terms of the $\zeta_{i}$ dynamics, for $i=2, \ldots, n$, are constructed using the "previous" observer variable $\zeta_{i-1}$ rather than the observed output $y$ (see the definition of $e_{i}$ in (11)). By contrast, in the classical observer (4), the relative degree between $y$ and the observation errors is always equal to one. Applied to linear systems, standard frequency-domain considerations can be used to conclude that, due to this relative degree property, the new observer behaves better than the classical one in terms of sensitivity of the observation error variables to high-frequency measurement noise. As shown in the numerical analysis presented in Section 5, the improvement in the sensitivity to highfrequency measurement noise is quite evident also in the nonlinear case. A proof of the result goes beyond the scope of the paper and it is not here provided.

\section{Nonlinear Separation Principle}

In this section we show that the observer presented in the previous section lends itself to be used in a nonlinear separation principle, in which the existence of a globally stabilizing state feedback control law, in conjunction with the observer (11), suffice to design an outputfeedback controller able to semi-globally stabilize the system. In this context the starting point is the existence of a state feedback control law that is formalised in the next assumption.

Assumption 3 There exists a $C^{1}$ function $\alpha^{\star}: \mathbb{R}^{n} \rightarrow$ $\mathbb{R}$ satisfying $\alpha^{\star}(0)=0$ such that the equilibrium $z=0$ of system (2) controlled by

$$
u=\alpha^{\star}(z)
$$

is globally asymptotically stable.

For the sake of brevity, let's denote the system (2) and (16) in the compact form

$$
\dot{z}=f\left(z, \alpha^{\star}(z)\right),
$$

in which $f(\cdot)=\operatorname{col}\left(f_{1}(\cdot), \ldots, f_{n}(\cdot)\right)$.

As the state $z$ is not available, the estimates of $z$ should be applied to replace the role of $z$ in the control. To prevent the presence of finite escape times for the closed-loop system, as originally proposed in [8], the actual control to be implemented on the system is

$$
u=\sigma_{R}\left(\alpha^{\star}\left(\hat{z}^{\prime}\right)\right)
$$

in which $\sigma_{R}(r)$ is a saturation function with the saturation level $R>0$ and where $\hat{z}^{\prime}$ is the state estimate provided by the observer (11) introduced in Section 3 .

By considering the change of coordinates (12), (14) and the resulting observer dynamics in (15), the closed loop system obtained by applying (17) reads as

$$
\begin{aligned}
& \dot{z}=f\left(z, \alpha^{\star}(z)\right)+\Delta_{1}(z, \varepsilon), \\
& \dot{\varepsilon}=\kappa M(t) \varepsilon+\bar{F}_{\kappa}(t)
\end{aligned}
$$

in which we have set

$$
\Delta_{1}(z, \varepsilon)=f\left(z, \sigma_{R}\left(\alpha^{\star}\left(z+\Delta_{\kappa} L_{1} \varepsilon\right)\right)\right)-f\left(z, \alpha^{\star}(z)\right) .
$$

It turns out that there exists a tuning of the saturation level $R$ and of the high-gain parameter $\kappa$ that make the origin of the closed loop system (18) asymptotically (and locally exponentially) stable with an arbitrary large domain of attraction. This is detailed in the following theorem whose proof, having in mind the claim of Theorem 2 , comes off-the-shelf from the arguments in [21].

Theorem 3 Consider system (1) having observability canonical form (2) in closed-loop with (11) and (17) and suppose Assumptions 1-3 hold. Let $\left(k_{i 1}, k_{i 2}\right), i=$ $1, \ldots, n-1$, be chosen according to Lemma 1 in such a way that the matrix $M(t)$ satisfies (10) for some positive definite and symmetric matrix $P$ and some positive $\lambda$, for all $t \geq 0$. Then, for any compact set $\mathcal{K} \in \mathbb{R}^{3 n-2}$, there exists a $R^{\star}>0$ and, for all $R \geq R^{\star}$, there exists $a \kappa^{\star} \geq 1$ such that, for all $\kappa>\kappa^{\star}$, the equilibrium $(z, \zeta)=(0,0)$ of the closed-loop system is asymptotically stable with a domain of attraction containing $\mathcal{K}$.

Remark: If the function $\alpha^{\star}: \mathbb{R}^{n} \rightarrow \mathbb{R}$ introduced in Assumption 3 is at least $C^{n}$, it is possible to combine the results in [9], [2] and [22] to obtain a different design. In particular, by following [9, Definition 3.1, Chapter 2], the system (1) can be immersed, by a suitable change of coordinates (which depends on $\left.u, \dot{u}, \ldots, u^{(n-1)}\right)$ into the so-called phase-variable representation

$$
\begin{aligned}
& \dot{s}_{1}=s_{2}, \ldots, \dot{s}_{n-1}=s_{n} \\
& \dot{s}_{n}=H\left(s_{1}, \ldots, s_{n}, u, \dot{u}, \ldots, u^{(n-1)}\right) .
\end{aligned}
$$

Then, if the change of coordinates is unique and globally defined, the observer proposed in [2] can be used to get an estimate of $\left(s_{1}, \ldots, s_{n}\right)$. For this, we need to consider $u, \dot{u}, \ldots, u^{(n-1)}$ as part of the state, as noted in [12, Section 9.6.1]. This can be done by extending the system 
with a chain of $n$ integrators on the control input $u$, and by designing a new control law for virtual input $u^{(n)}$. As showed for instance in $[22,12]$, the design relies on backstepping technique. In this different approach, the design of the observer turns out to be much more simpler, but at the price of asking the existence (not always guaranteed) of a global change of coordinates (which may be very hard to compute for the general class of systems (1)) and the application of $n$-steps of backstepping (which involves in general a considerable computational effort).

\section{Simulation example}

We consider a single-link robot arm system (see [12, Section 4.10]) described by

$$
\begin{aligned}
\dot{z}_{1} & =z_{2} \\
\dot{z}_{2} & =\frac{\bar{K}}{J_{2} N} z_{3}-\frac{F_{2}}{J_{2}} z_{2}-\frac{\bar{K}}{J_{2}} z_{1}-\frac{m g d}{J_{2}} \cos z_{1} \\
\dot{z}_{3} & =z_{4} \\
\dot{z}_{4} & =\frac{1}{J_{1}} u+\frac{\bar{K}}{J_{1} N} z_{1}-\frac{\bar{K}}{J_{2} N} z_{3}-\frac{F_{1}}{J_{1}} z_{4} \\
y & =z_{1}
\end{aligned}
$$

where $J_{1}$ and $J_{2}$ represent the inertias of the actuator shaft and the link respectively, $\bar{K}$ denotes the elasticity constant of the joint elastic coupling, $N$ is the transmission gear ration, $m$ is the mass, $g$ is the gravity acceleration, $d$ is the position of the center of the gravity of the link, and $F_{1}$ and $F_{2}$ are viscous friction coefficients. We are interested in stabilizing by output feedback the equilibrium $z=(0,0, m g d N / \bar{K}, 0)$ of (19) by following the result of Theorem 3 . It can be verified that system (19) satisfies Assumptions 1 and 2 and therefore we can design an observer of the form (11) by following Lemma 1 and Theorem 2. Moreover, note that the technique proposed in [2] cannot be applied directly in this framework without an appropriate coordinate transformation. Assumption 3 is verified by the following control law

$$
\begin{aligned}
u=\frac{m g d J_{1}}{J_{2} N}-\frac{J_{1} J_{2} N}{\bar{K}}\left[L^{4} c_{1} z_{1}+L^{3} c_{2} z_{2}+\right. \\
\left.L^{2} c_{3}\left(\frac{\bar{K}}{J_{2} N} z_{3}-\frac{m g d}{J_{2}}\right)+L c_{4} \frac{\bar{K}}{J_{2} N} z_{4}\right],
\end{aligned}
$$

where $c_{i}, i=1, \ldots, 4$ are positive coefficients to be chosen and $L>0$ is a constant to be chosen large enough.

The values of the physical parameters of (19) have been taken as $F_{1}=0.1, F_{2}=0.15, J_{1}=0.15, J_{2}=0.2$, $\bar{K}=0.4 N=2, m=0.8, g=9.81$ and $d=0.6$. The parameters of the control law (20) are chosen as $c_{1}=4$, $c_{2}=7.91, c_{3}=6.026, c_{4}=1.716, L=3$, and the control law is implemented by following (17) with $R=200$. The observer is implemented as (11) where the coefficients are chosen as

$$
\begin{array}{lll}
k_{11}=2.5, & k_{21}=2.5, & k_{31}=2.5, \\
k_{12}=4.6, & k_{22}=1.533, & k_{32}=0.511
\end{array}
$$

and $\kappa=250$. The initial conditions are set as $z=$ $(0.5,0,0,0)$ for the plant and $\zeta=0$ for the observer. In the simulation we considered the case when the measured output is given by

$$
y=z_{1}+\nu(t), \quad \nu=\varrho \sin (\omega t),
$$

with $\varrho=0.002$ and $\omega=3000$. Figure 1 shows the behaviour of the state $z$ of the closed loop system (19), (20) with the observer (11) when there is no measurement noise and when the measurement noise is present.

We are also interested in the performances of the proposed observer structure in presence of highfrequency measurement noise to see if there are improvements in the sensitivity with respect to a standard highgain observer (4). As a comparison, the coefficients of (4) are chosen as $\left(k_{1}, k_{2}, k_{3}, k_{4}\right)=(5,9.35,7.75,2.4024)$ and the initial condition is set as $\hat{z}(0)=0$. The high-gain parameter is chosen as $\kappa=202$ in order to practically match the convergence rate of the two observers. In this simulations we considered the case in which the system is controlled by state feedback and we are using the two observers only to get an estimate of the state $z$ of the plant. As in [2], it can be verified that new observer provides better properties with respect to the standard high-gain construction (4) in terms of sensitivity of the estimation errors to high-frequency measurement noise, despite the gain chosen for the new observer is higher than the one used for the standard high-gain observer with the same convergence rate. This is shown in Table 1 in which the normalized asymptotic magnitude estimate errors are shown for the two observers.

\begin{aligned} & \hline $\begin{array}{c}\text { Standard High Gain } \\ \text { Observer } \hat{z}\end{array} \multicolumn{1}{c}{\begin{array}{c}\text { Modified } \\ \text { Observer } \hat{z}^{\prime}=L_{1} \zeta\end{array}} \\ &$\hline$\left|z_{1}-\hat{z}_{1}\right|_{a}^{\varrho} \simeq 3.3 \cdot 10^{-1}\left|z_{1}-\hat{z}_{1}^{\prime}\right|_{a}^{\varrho} \simeq 2.1 \cdot 10^{-1} \\ &\left|z_{2}-\hat{z}_{2}\right|_{a}^{\varrho} \simeq 1.3 \cdot 10^{2}\left|z_{2}-\hat{z}_{2}^{\prime}\right|_{a}^{\varrho} \simeq 20 \\ &\left|z_{3}-\hat{z}_{3}\right|_{a}^{\varrho} \simeq 2.1 \cdot 10^{4}\left|z_{3}-\hat{z}_{3}^{\prime}\right|_{a}^{\varrho} \simeq 6.2 \cdot 10^{2} \\ &\left|z_{4}-\hat{z}_{4}\right|_{a}^{\varrho} \simeq 1.3 \cdot 10^{6}\left|z_{4}-\hat{z}_{4}^{\prime}\right|_{a}^{\varrho} \simeq 3.2 \cdot 10^{4} \\ &$\hline\end{aligned}

Table 1: Normalized asymptotic errors in presence of noise. $|x(t)|_{a}^{\varrho}$ denotes the asymptotic norm normalized with respect to $\varrho$, i.e. $|x(t)|_{a}^{\varrho}=\limsup _{t \rightarrow \infty}|x(t)| / \varrho$.

\section{Conclusion}

In this paper we presented a new high-gain observer for nonlinear systems which are uniform observable (ac- 

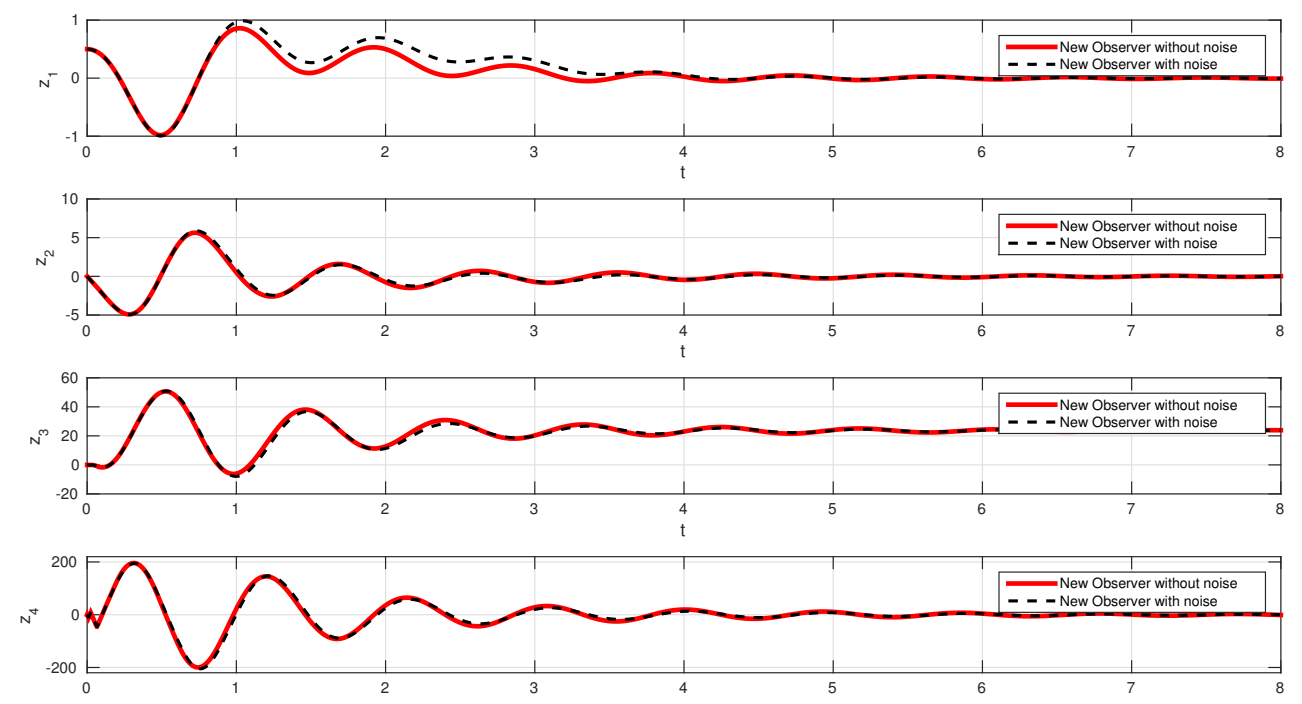

Fig. 1. State $z$ of the closed loop system (19), (20) when using the observer (11) with and without measurement noise

cording to the definition in [9]). The strength of the new observer relies in the fact that the power of the high-gain parameter is always 2 regardless the dimension of the observed system. This feature makes always possible its implementability even when the dimension of the system is very large. We showed that the observer can be also used in output feedback stabilisation within a canonical nonlinear separation principle. The result is inspired by the observer design technique recently proposed in [2] for the class of systems that are transformable in the canonical observability form. The new observer does not present any benefit or drawbacks in terms of peaking phenomenon. However, the recent technique introduced in [3] could be applied to this framework in order to overcome the peaking phenomenon. Finally, as already noted in [2], we observe that the proposed structure provides better results with respect to the standard highgain observer in terms of sensitivity to high-frequency measurement noise. This is not formally proved but only verified through simulations. A complete characterization of the behaviour of the observer in presence of noise is under study.

\section{A The proof of Lemma 1}

The idea of the proof is to iterate a small-gain theorem by starting from the block of $M(t)$ on the bottom. For convenience, we first recursively set matrices $M_{i}(t) \in \mathbb{R}^{(2 i) \times(2 i)}$ as

$$
\begin{aligned}
M_{1}(t) & :=E_{n-1}(t) \\
M_{i+1}(t) & :=\left(\begin{array}{cc}
E_{n-i-1}(t) & \bar{N}_{n-i}(t) \\
\bar{Q}_{n-i} & M_{i}(t)
\end{array}\right), \quad i=1, \ldots, n-2,
\end{aligned}
$$

where $\bar{N}_{n-i}(t) \in \mathbb{R}^{2 \times 2 i}$ and $\bar{Q}_{n-i} \in \mathbb{R}^{2 i \times 2}$ are defined as

$$
\begin{aligned}
\bar{N}_{n-1}(t) & :=N_{n-1}(t), \quad \bar{N}_{n-i}(t):=\left(\begin{array}{lll}
N_{n-i}(t) & 0 \ldots 0
\end{array}\right), \\
\bar{Q}_{n-1} & :=Q_{n-1}, \quad \bar{Q}_{n-i}:=\left(\begin{array}{llll}
Q_{n-i}^{\top} & 0 \ldots 0
\end{array}\right)^{\top} .
\end{aligned}
$$

The proof of Lemma 1 immediately comes by the following two lemmas.

Lemma 2 Consider the matrix $M_{1}(t)$. There exist coefficients $k_{n-1,1}$ and $k_{n-1,2}$ and a positive definite symmetric matrix $P_{1}$ such that

$$
P_{1} M_{1}(t)+M_{1}^{\top}(t) P_{1} \leq-\lambda_{1} I
$$

for some positive constant $\lambda_{1}$.

Proof. Consider the system

$$
\dot{\xi}_{1}=E_{n-1}(t) \xi_{1}
$$

in which $\xi_{1}=\operatorname{col}\left(\xi_{11}, \xi_{12}\right) \in \mathbb{R}^{2}$. Let $\Theta(r)$ be the matrix having the form

$$
\Theta(r)=\left(\begin{array}{cc}
r & 0 \\
-r & 1
\end{array}\right)
$$

for all $r \in \mathbb{R}$, and then consider the following change of variables

$\eta_{1}=\Theta\left(\gamma_{1}\right) \xi_{1} \quad$ i.e. $\quad \eta_{11}=\gamma_{1} \xi_{11}, \quad \eta_{12}=\xi_{12}-\gamma_{1} \xi_{11}$ 
with $\gamma_{1}>0$ to be chosen. System (A.1) in the new coordinates can be rewritten as ${ }^{2}$

$$
\begin{gathered}
\dot{\eta}_{11}=-\left[k_{n-1,1} b_{n-1,1}-\gamma_{1} a_{n-1}\right] \eta_{11}+\gamma_{1} a_{n-1} \eta_{12} \\
\dot{\eta}_{12}=-\left[\left(\gamma_{1}^{-1} k_{n-1,2}-k_{n-1,1}\right) b_{n-1,1}+\gamma_{1} a_{n-1}\right] \eta_{11} \\
-\gamma_{1} a_{n-1} \eta_{12} .
\end{gathered}
$$

By taking $k_{n-1,2}=\gamma_{1} k_{n-1,1}$, we have the system

$$
\begin{aligned}
& \dot{\eta}_{11}=-\left(k_{n-1,1} b_{n-1,1}-\gamma_{1} a_{n-1}\right) \eta_{11}+\gamma_{1} a_{n-1} \eta_{12} \\
& \dot{\eta}_{12}=-\gamma_{1} a_{n-1} \eta_{11}-\gamma_{1} a_{n-1} \eta_{12} .
\end{aligned}
$$

Now choose the Lyapunov function

$$
V_{1}=\left|\eta_{1}\right|^{2}=\xi_{1} \Theta\left(\gamma_{1}\right)^{\top} \Theta\left(\gamma_{1}\right) \xi_{1}
$$

whose time derivative is given by

$$
\dot{V}_{1}=-2\left(k_{n-1,1} b_{n-1,1}-\gamma_{1} a_{n-1}\right) \eta_{11}^{2}-2 \gamma_{1} a_{n-1} \eta_{12}^{2}
$$

By coming back in the $\xi_{1}$-coordinates and by using Young's inequality, the above equality can be rewritten as

$$
\begin{aligned}
\dot{V}_{1} & \leq-2 \gamma_{1}^{2}\left(k_{n-1,1} b_{n-1,1}-2 \gamma_{1} a_{n-1}\right) \xi_{11}^{2}-\gamma_{1} a_{n-1} \xi_{12}^{2} \\
& \leq-2 \gamma_{1}^{2}\left(k_{n-1,1} \alpha-2 \gamma_{1} \beta\right) \xi_{11}^{2}-\gamma_{1} \alpha \xi_{12}^{2}
\end{aligned}
$$

Given any positive $\gamma_{1}$, and choosing $k_{n-1,1}>2 \gamma_{1} \frac{\beta}{\alpha}$, we can conclude that $\dot{V}_{1} \leq-\lambda_{1}\left|\xi_{1}\right|^{2}$ with $\lambda_{1}=$ $\min \left\{2 \gamma_{1}^{2}\left(k_{n-1,1} \alpha-2 \gamma_{1} \beta\right), \gamma_{1} \alpha\right\}$. Namely, given $P_{1}=$ $\Theta\left(\gamma_{1}\right)^{\top} \Theta\left(\gamma_{1}\right)$, the inequality $P_{1} M_{1}(t)+M_{1}(t)^{\top} P_{1} \leq$ $-\lambda_{1} I$ holds, which completes the proof of Lemma 2 .

Lemma 3 Assume there exist a symmetric positive definite matrix $P_{i}$ and a positive constant $\lambda_{i}$ such that $P_{i} M_{i}(t)+M_{i}(t)^{\top} P_{i} \leq-\lambda_{i} I$. Then there exist coefficients $k_{n-i-1,1}$ and $\bar{k}_{n-i-1,2}$ and a positive definite symmetric matrix $P_{i+1}$ such that

$P_{i+1} M_{i+1}(t)+M_{i+1}^{\top}(t) P_{i+1} \leq-\lambda_{i+1} I, \quad 1 \leq i \leq n-2$ for some positive constant $\lambda_{i+1}$.

Proof. Consider the system

$$
\begin{aligned}
\dot{\xi}_{i+1} & =E_{n-i-1}(t) \xi_{i+1}+\bar{N}_{n-i}(t) \chi_{i} \\
\dot{\chi}_{i} & =M_{i}(t) \chi_{i}+\bar{Q}_{n-i} \xi_{i+1}
\end{aligned}
$$

\footnotetext{
${ }^{2}$ From now on we omit the time-dependence in the variables for the purpose of compactness.
}

where $\xi_{i+1}=\operatorname{col}\left(\xi_{i+1,1}, \xi_{i+1,2}\right) \in \mathbb{R}^{2}$ and $\chi_{i}=$ $\operatorname{col}\left(\xi_{1}, \ldots, \xi_{i}\right) \in \mathbb{R}^{2 i}$. Let's make the following linear coordinate change for the state $\xi_{i+1}$ in (A.3)

$$
\eta_{i+1}:=\operatorname{col}\left(\eta_{i+1,1}, \eta_{i+1,2}\right)=\Theta\left(\gamma_{i+1}\right) \xi_{i+1}
$$

where $\Theta\left(\gamma_{i+1}\right)$ has the form (A.2) and $\gamma_{i+1}$ is a positive constant to be chosen. The system (A.3) in the new coordinates can be rewritten as ${ }^{3}$

$$
\begin{gathered}
\dot{\eta}_{i+1,1}=-\left[k_{n-i-1,1} b_{n-i-1,1}-\gamma_{i+1} a_{n-i-1}\right] \eta_{i+1,1} \\
\quad+\gamma_{i+1} a_{n-i-1} \eta_{i+1,2} \\
\dot{\eta}_{i+1,2}=-\left[\left(\gamma_{i+1}^{-1} k_{n-i-1,2}-k_{n-i-1,1}\right) b_{n-i-1,1}\right. \\
\left.+\gamma_{i+1} a_{n-i-1}\right] \eta_{i+1,1}-\gamma_{i+1} a_{n-i-1} \eta_{i+1,2} \\
\quad+\bar{N}_{n-i} \chi_{i} \\
\dot{\chi}_{i}=M_{i} \chi_{i}+\Gamma_{i}\left(\eta_{i+1,2}+\eta_{i+1,1}\right)
\end{gathered}
$$

where $\Gamma_{i}=\operatorname{col}\left(k_{n-i, 1}, k_{n-i, 2}, 0, \ldots, 0\right)$. By taking $k_{n-i-1,2}=\gamma_{i+1} k_{n-i-1,1}$, we get

$$
\begin{gathered}
\dot{\eta}_{i+1,1}=-\left[k_{n-i-1,1} b_{n-i-1,1}-\gamma_{i+1} a_{n-i-1}\right] \eta_{i+1,1} \\
+\gamma_{i+1} a_{n-i-1} \eta_{i+1,2} \\
\dot{\eta}_{i+1,2}=-\gamma_{i+1} a_{n-i-1} \eta_{i+1,1}-\gamma_{i+1} a_{n-i-1} \eta_{i+1,2} \\
\quad+\bar{N}_{n-i} \chi_{i} \\
\dot{\chi}_{i}=M_{i} \chi_{i}+\Gamma_{i}\left(\eta_{i+1,2}+\eta_{i+1,1}\right) .
\end{gathered}
$$

Consider now the positive definite function $V_{i}=\chi_{i}^{\top} P_{i} \chi_{i}$, whose time derivative is given by

$$
\begin{aligned}
\dot{V}_{i} & =2 \chi_{i}^{\top} P_{i}\left[M_{i}(t) \chi_{i}+\Gamma_{i}\left(\eta_{i+1,2}+\eta_{i+1,1}\right)\right] \\
& \leq-\lambda_{i}\left|\chi_{i}\right|^{2}+2 \chi_{i}^{\top} P_{i} \Gamma_{i} \xi_{i+1,2} \\
& \leq-\frac{1}{2} \lambda_{i}\left|\chi_{i}\right|^{2}+\delta_{1} \xi_{i+1,2}^{2}
\end{aligned}
$$

for some positive $\delta_{1}$, independent of $\gamma_{i+1}$ and $k_{n-i-1,1}$. Furthermore, consider the positive definite function

$$
W_{i+1}=\left|\eta_{i+1}\right|^{2}=\xi_{i+1} \Theta\left(\gamma_{i+1}\right)^{\top} \Theta\left(\gamma_{i+1}\right) \xi_{i+1}
$$

\footnotetext{
3 Again, from now on we omit the time-dependence in the variables for the purpose of compactness.
} 
whose time derivative is given by

$$
\begin{aligned}
\dot{W}_{i+1}=- & 2\left[k_{n-i-1,1} b_{n-i-1,1}-\gamma_{i+1} a_{n-i-1}\right] \eta_{i+1,1}^{2} \\
& -2 \gamma_{i+1} a_{n-i-1} \eta_{i+1,2}^{2}+2 \eta_{i+1,2} \bar{N}_{n-i} \chi_{i} \\
\leq & -2\left[k_{n-i-1,1} b_{n-i-1,1}-\gamma_{i+1} a_{n-i-1}\right] \eta_{i+1,1}^{2} \\
& -\gamma_{i+1} a_{n-i-1} \eta_{i+1,2}^{2}+\frac{\beta^{2}}{\alpha \gamma_{i+1}}\left|\chi_{i}\right|^{2} \\
\leq & -2 \gamma_{i+1}^{2}\left[k_{n-i-1,1} b_{n-i-1,1}-2 \gamma_{i+1} a_{n-i-1}\right] \xi_{i+1,1}^{2} \\
& \quad-\frac{3}{4} \gamma_{i+1} a_{n-i-1} \xi_{i+1,2}^{2}+\frac{\beta^{2}}{\alpha \gamma_{i+1}}\left|\chi_{i}\right|^{2} \\
\leq & -2 \gamma_{i+1}^{2}\left[k_{n-i-1,1} \alpha-2 \gamma_{i+1} \beta\right] \xi_{i+1,1}^{2} \\
& \quad-\frac{3}{4} \gamma_{i+1} \alpha \xi_{i+1,2}^{2}+\frac{\beta^{2}}{\alpha \gamma_{i+1}}\left|\chi_{i}\right|^{2} .
\end{aligned}
$$

Then consider the Lyapunov function $V_{i}+W_{i+1}$. By choosing $\gamma_{i+1}$ such that $\gamma_{i+1}=\max \left\{\frac{2 \delta_{1}}{\alpha}, \frac{4 \beta^{2}}{\lambda_{i} \alpha}\right\}$ and $k_{n-i-1,1}$ satisfying $k_{n-i-1,1}>2 \gamma_{i+1} \beta / \alpha$, we get

$$
\begin{aligned}
\dot{V}_{i}+\dot{W}_{i+1} \leq & -\frac{\lambda_{i}}{4}\left|\chi_{i}\right|^{2}-\frac{1}{4} \gamma_{i+1} \alpha \xi_{i+1,2}^{2} \\
& -2 \gamma_{i+1}^{2}\left[k_{n-i-1,1} \alpha-2 \gamma_{i+1} \beta\right] \xi_{i+1,1}^{2}
\end{aligned}
$$

Now set $\chi_{i+1}=\operatorname{col}\left(\xi_{i+1}, \chi_{i}\right)$ and

$$
P_{i+1}=\operatorname{blkdiag}\left(\Theta\left(\gamma_{i+1}\right)^{\top} \Theta\left(\gamma_{i+1}\right), P_{i}\right),
$$

and consider the positive definite function $V_{i+1,1}=$ $\chi_{i+1}^{\top} P_{i+1} \chi_{i+1}$. Its time derivative satisfies

$$
\dot{V}_{i+1} \leq-\lambda_{i+1}\left|\chi_{i+1}\right|^{2}
$$

in which

$\lambda_{i+1}=\min \left\{\frac{\lambda_{i}}{4}, 2 \gamma_{i+1}^{2}\left(k_{n-i-1,1} \alpha-2 \gamma_{i+1} \beta\right), \frac{1}{4} \gamma_{i+1} \alpha\right\}$.

That is,

$$
P_{i+1} M_{i+1}(t)+M_{i+1}(t)^{\top} P_{i+1} \leq-\lambda_{i+1} I
$$

which completes the proof of Lemma 3 .

\section{References}

[1] J. H. Ahrens and H. K. Khalil, "High-gain observer in the presence of measurement noise: a switched-gain approach", Automatica vol. 45, pp. 936-943, 2009.

[2] D. Astolfi and L. Marconi, "A high-gain nonlinear observer with limited gain power", IEEE Trans. Automatic Control, vol. 60 (11), pp. 3059-3064, 2015.

[3] D. Astolfi, L. Marconi and A. Teel, "Low-power peakingfree high-gain observers for nonlinear systems", 2016 European Control Conference, 2016 (Accepted).
[4] A. A. Ball and H. K. Khalil, "High-gain observer tracking performance in the presence of measurement noise", American Control Conference, pp. 4626-4627, 2009.

[5] G. Besançon, Nonlinear observers and applications. Springer Verlag, vol. 363, 2007.

[6] F. Esfandiari and H.K. Khalil, "Observer-based design of uncertain systems: recovering state feed- back robustness under matching conditions", In Proc. Allerton Conf., pages 97-106, Monticello, IL, September 1987.

[7] F. Esfandiari and H.K. Khalil. Output feedback stabilization of fully linearizable systems. International Journal of Control, vol.56, no.5, pp. 1007-1037, 1992.

[8] J.P. Gauthier and I. Kupka, "Deterministic observation theory and applications", Cambridge University Press, 2004.

[9] J.P. Gauthier and G. Bornard, "Observability for any $u(t)$ of a Class of Nonlinear Systems", IEEE Trans. Automatic Control, vol. 26, no. 4, pp. 922-926, 1981.

[10] J. Gauthier, H. Hammouri and S. Othman, "A simple observer for nonlinear systems aplication to bioreactors", IEEE Trans. Automat. Contr., vol.37, no.6, pp. 875-880, 1992.

[11] A. Isidori, "Nonlinear Control Systems", New York: Springer, 1995.

[12] A. Isidori, "Nonlinear Control Systems II", New York: Springer, 1999

[13] H. K. Khalil and F. Esfandiari "Semiglobal stabilization of a class of nonlinear systems using output feedback", IEEE Trans. Automat. Control, vol. 38, pp. 1412-1415, 1993.

[14] H. K. Khalil, "High-gain Observers in Nonlinear Feedback Control", in Int. Conf. Contr. Automat. Syst., pp. 47 - 57, Seoul, Korea 2008.

[15] H. K. Khalil and L. Praly, "High-gain Observers in Nonlinear Feedback Control", in Int. J. of Robust and Nonlinear Control, vol. 24, issue 6, pp. 993-1015, 2014.

[16] L. Marconi, L. Praly and A. Isidori. Output stabilization via nonlinear Luenberger observers. SIAM Journal on Control and Optimazation, vol.45, no.5, pp.2277-2298, 2007.

[17] R. Marino and P. Tomei, "Dynamic output feedback linearization and global stabilization", System Control Lett., Vol. 17, pp. 115-121, 1991.

[18] H. Shim and J. H.Seo. "Non-linear output feedback stabilization on a bounded region of attraction," International Journal of Control, vol.73, no.73, pp. 416-426, 2000

[19] A. Teel and L. Praly, "Global stabilizability and observability imply semi-global stabilizability by output feedback", Systems E Control Letters, vol. 22, pp. 313-325, 1994.

[20] A. Teel and L. Praly, "Tools for semiglobal stabilization by partial state and output feedback", SIAM Journal on Control and Optimization, vol. 33, no.5, pp. 1443-1488, 1995.

[21] A. Tornambé, "High-gain observers for non-linear systems", Int. J. of Systems Science, vol. 23, no.9, pp. 1475-1489, 1992.

[22] L. K. Vasiljevic and H. K. Khalil, "Differentiation with highgain observers the presence of measurement noise", IEEE 45 th Conference on Decision and Control, pp. 4717-4722, December 2006. 